If for $x$ in the given series we substitute $\pi+\epsilon$ we have, omitting the factor 2 ,

$$
-y=\sin \epsilon+\frac{1}{2} \sin 2 \epsilon+\frac{1}{3} \sin 3 \epsilon++\frac{1}{n} \sin n \epsilon+\ldots
$$

This series increases with $n$ until $n \epsilon=\pi$. Suppose, therefore, $\epsilon=k \frac{\pi}{n}$, where $k$ is a small fraction. The series will now be nearly equal to $n \epsilon=k \pi$, a finite quantity even if $n=\infty$.

Hence the value of $y$ in the immediate vicinity of $x=\pi$ is not an isolated point $y=0$, but a straight line $-y=n x$.

The same result is obtained by differentiation, which gives

$$
\frac{d y}{d x}=\cos x-\cos 2 x+\cos 3 x-\ldots
$$

putting $x=\pi+\epsilon$ this becomes

$$
-\frac{d y}{d x}=\cos \epsilon+\cos 2 \epsilon+\cos 3 \epsilon+\ldots+\cos n \epsilon+
$$

which is nearly equal to $n$ for values of $n \epsilon$ less than $k \pi$.

It is difficult to see the meaning of the tangent if $y$ were an isolated point.

Albert A. Michelson.

The University of Chicago Ryerson Physical Laboratory, September 6

\section{Helium in the Atmosphere.}

C. FRIEDläNDER and H. Kayser have independently claimed to have found helium in the atmosphere. On examination of some photographs of the spectrum of neon I have identified six of the principal lines of helium, which thus establishes beyond question the presence of this gas in the air. The amoun present in the neon it is, of course, impossible to estimate, but the green line (wave-length 5016 ) is the brightest, as would be expected from the low pressure of the helium in the neon.

E. C. C. BALY.

University College, London, Gower Street, W.C. September 28.

THE discovery of helium lines in the spectrum of neon, by Mr. E. C. C. Baly, will necessitate a modification of the views we have expressed in our communication to the British Association at Bristol. We there estimated the density of neon at 9.6 , allowing for the presence of a certain proportion of argon unavoidably left in the neon. As it contains helium, however, this is probably an under-estimate. It is unfortunately not possible to form any estimate of the amount of helium mixed with the neon from the relative intensity of spectrum lines, as has been already shown by Dr. Collie and one of us; we do not despair, however, of removing a large part, if not all of this helium, by taking advantage of the greater solubility of neon than helium in liquid oxygen.

The presence of helium, however, in no way alters our view as to the position of neon in the periodic table. The number $9^{\circ} 6$ implies an atomic weight of $19^{\prime} 2$; and a somewhat higher atomic weight would even better suit a position between fluorine, 19, and sodium, 23 . WILLIAM RAMSAY.

University College, London, MORRIS W. TRAVERS.

Gower-street, W.C., September 28.

\section{Chance or Vitalism?}

I AM glad to see that Prof. Karl Pearson has called attention to Prof. Japp's address at Bristol. Only that one does not like to criticise adversely a presidential address, I would at the time have pointed out the weakness in the argument that Prof. Pearson criticises. He does not go nearly so far in this criticism as the circumstances warrant. It is conceded that right-and left-handed crystals of quite sensible size are produced sufficiently separated to be seen and handled as separate crystals. Now assuming, what there is every reason otherwise to think quite probable, that life started from some few centres, the chances are, not that it was equally divided between right- and left-handed forms, but that one or other of these forms preponderated. In fact, if life started from a single centre, it must have been either right- or left-handed. Hence the fact adduced only shows, what was otherwise very probable, that life started from a small number of origins, possibly only one.

NO. I 5 IO, VOL. 58 ]
Another reason for either a right- or left-handed structure in living organisms on the earth, and one which diminishes the force of the foregoing argument for a small number of origins, is that it probably started either in the northern or in the southern hemisphere, and in either case the rotation of the sun in the heavens may be a sufficient cause for a right- or left-handed structure in an organism growing under its influence.

Trinity College, Dublin, September 27.

Geo. Fras. FitzGerai.d.

IN his presidential address to Section B of the British Association, Prof. Japp argues the necessity of supposing a "directive force," or intelligence, to have guided the formation of the first asymmetric substance. "Vitalism," which at one time was supposed to regulate the physiology and even the mechanics of organised beings, has passed more and more from the foreground, till, in the vision of some it remains only as a point in the vast distance of time at the origin of life. Is it to disappear altogether?

A sensible quantity of a mixture of enantiomorphs contains an enormous number of molecules. Chance determines the relative proportion present of right- and left-handed forms. Each molecule, having resulted from the action of symmetric forces, has an even chance of being of one or the other. Hence, the improbability of there being present a great preponderance of one form over the other is so great, that it is inconceivable that an optically active solution could result. To the above contention of Prof. Japp, the reply is made by Prof. Karl Pearson, in NATURE of September 22, that a chance result, however improbable, will occur, if sufficient opportunity be allowed. He postulates the vast ages of the earth's history. May we not, however, invoke chance to deal with masses instead of molecules, and thus perhaps substitute weeks for ages?

Let us consider a solution, in which the numbers of right-and left-handed molecules are very approximately equal, and which is consequently optically inactive. In the slow evaporation of the solvent, the right- and left-handed nuclei, about which the substance crystallises, will most probably be evenly distributed. Their number will be extremely small in comparison with that of the molecules, and, as chance determines their distribution, it is not so highly improbable-it is at least conceivable-that the crystals will be unevenly grouped. Suppose such to take place and a partial re-solution, roughly in the lines of the distribution of the two varieties of crystals-a not very improbable event-and we have an optically active solution. Chance has here acted the part played by organised matter in the person of M. Pasteur, by selecting and rejecting the oppositely formed crystals.

Is it yet possible to deny that the first ancestor of læevorotatory protein could have been built up from an asymmetric substance, separated in some such way as the above, by the play of chance upon the natural working of symmetric forces? Clement O. Bartrum.

I7 Denning Road, Hampstead, N.W., September 24.

\section{The Moon's Course.}

MAY I refer Sir S. Wilks to the simple and beautifully written autobiography of James Ferguson, F.R.S., self-taught mechanician and astronomer? I will quote a passage.

"Soon afterwards" (the previous date was I 743) "it appeared to me, that although the moon goes round the earth, and that the sun is far on the outside of the moon's orbit-yet the moon's motion must be in a line-that is, always concave toward the sun: and upon making a delineation representing her absolute path in the heavens-I found it to be really so. I then made a simple machine for delineating both her path, and the earth's, on a long paper laid on the floor. I carried the machine and the delineation to the late Martin Folkes, Esquire, President of the Royal Society, on a Thursday afternoon. He expressed great satisfaction at seeing it, as it was a new discovery, and took me that evening to the Royal Society, where I showed the delineation and the method of doing it. When the business of the Society was over, one of the members desired me to dine with him the next Saturday at Hackney, telling me that his name was Ellicott, and that he was a watchmaker. I accordingly went and was kindly received by Mr. Ellicott, who then showed me the very same kind of delineation and part of the 